\title{
LEADING TERMS IN THE HEAT INVARIANTS
}

\author{
THOMAS P. BRANSON, PETER B. GILKEY, AND BENT ØRSTED
}

(Communicated by Palle E. T. Jorgensen)

\begin{abstract}
Let $D$ be a second-order differential operator with leading symbol given by the metric tensor on a compact Riemannian manifold. The asymptotics of the heat kernel based on $D$ are given by homogeneous, invariant, local formulas. Within the set of allowable expressions of a given homogeneity there is a filtration by degree, in which elements of the smallest class have the highest degree. Modulo quadratic terms, the linear terms integrate to zero, and thus do not contribute to the asymptotics of the $L^{2}$ trace of the heat operator; that is, to the asymptotics of the spectrum. We give relations between the linear and quadratic terms, and use these to compute the heat invariants modulo cubic terms. In the case of the scalar Laplacian, qualitative aspects of this formula have been crucial in the work of Osgood, Phillips, and Sarnak and of Brooks, Chang, Perry, and Yang on compactness problems for isospectral sets of metrics modulo gauge equivalence in dimensions 2 and 3 .
\end{abstract}

\section{INTRODUCTION}

Let $(M, g)$ be a compact, $m$-dimensional Riemannian manifold without boundary. Let $R, \rho$, and $\tau$ be the Riemann, Ricci, and scalar curvatures, normalized so that on the standard sphere, $\rho=(m-1) g$ and $\tau=m(m-1)$. Let $D$ be a second-order differential operator on a vector bundle $V$. We assume that the leading symbol of $D$ is given by the metric tensor so that locally,

$$
D=-\left(g^{i j} \partial_{i} \partial_{j}+p^{i} \partial_{i}+q\right) \text {, }
$$

where $p$ is a local section of $T M \otimes \operatorname{End} V$ and $q$ is a local section of End $V$. Here and below, we adopt the convention of summing over repeated indices.

It will be convenient to express the total symbol of $D$ in a more invariant way. Given a connection $\nabla$ on $V$, we can form the Bochner, or reduced Laplacian

$$
\Delta=\Delta_{\nabla}=-g^{i j} \nabla_{i} \nabla_{j} .
$$

If $E$ is any bundle endomorphism of $V$, the leading symbol of the operator $\Delta_{\nabla}-E$ is, of course, given by the metric tensor. Conversely, given an operator

Received by the editors September 5, 1989.

1980 Mathematics Subject Classification (1985 Revision). Primary 47F05.

The first author was partially supported by a University of Iowa Faculty Scholar Award and by the Danish Research Academy. The first and third authors were partially supported by a NATO Collaborative Grant. The second author was partially supported by the NSF and NSA. 
$D$ which is locally of the form (1.1), there exists a unique pair $(\nabla, E)$ with $D=\Delta_{\nabla}-E$. Indeed, if $\Gamma_{i j}{ }^{k}$ are the Christoffel symbols of the Levi-Civita connection of $g$ and $\omega$ is the local connection one-form of $\nabla$, we just need to set

$$
\begin{aligned}
\omega_{l} & =\frac{1}{2} g_{i l}\left(p^{i}+g^{j k} \Gamma_{j k}{ }^{i}\right), \\
E & =q-g^{i j}\left(\partial_{j} \omega_{i}+\omega_{i} \omega_{j}\right)+g^{j k} \omega_{i} \Gamma_{j k}{ }^{i} .
\end{aligned}
$$

(See $[\mathrm{G} 1, \S 1]$ for details.) The components of the curvature two-form $\Omega$ of the connection $\nabla$ are

$$
\Omega_{i j}=\partial_{i} \omega_{j}-\partial_{j} \omega_{i}+\left[\omega_{i}, \omega_{j}\right]
$$

For $t>0$, the heat operator $e^{-t D}$ is trace class with smooth kernel $H(t, x, y)$. The diagonal values of $H$ admit a small-time asymptotic expansion

$$
H(t, x, x) \sim(4 \pi t)^{-m / 2} \sum_{n=0}^{\infty} e_{n}(x, D) t^{n}, \quad t \downarrow 0,
$$

where $e_{n}(x, D)$ are local endomorphism-valued invariants of $D$. (See [G2, $\S 1]$ for details.) The $e_{n}(x, D)$ are built universally and polynomially from the metric tensor, its inverse, and covariant derivatives of $R, \Omega$, and $E$. By $\mathrm{H}$. Weyl's work on the invariants of the orthogonal group, these polynomials can be formed using only tensor product and contraction of tensor arguments (indices). The $e_{n}(x, D)$ satisfy a certain homogeneity condition which can be expressed in many ways. Most relevant to our needs is the following formulation: if $Q$ is a monomial term of $e_{n}(x, D)$ of degree $\left(k_{R}, k_{\Omega}, k_{E}\right)$ in $(R, \Omega, E)$, and if $k_{\nabla}$ explicit covariant derivatives appear in $Q$, then

$$
2\left(k_{R}+k_{\Omega}+k_{E}\right)+k_{\nabla}=2 n .
$$

(Of course, an occurrence of $\rho$ or $\tau$ is counted as an occurrence of $R$.)

Let $\mathscr{P}_{2 n}$ be the vector space of all such $2 n$-homogeneous invariant endomorphisms; $e_{n}(x, D) \in \mathscr{P}_{2 n}$. In principle, this space depends on the dimension $m$ of the underlying manifold and the fiber dimension of $V$, but we shall suppress these complications as they do not appear in the Weyl calculus; again see [G1, §1] for details. $\mathscr{P}_{2 n}$ can be filtered as follows. Let $\mathscr{P}_{2 n, \ell}$ be the vector subspace of all endomorphisms which can be expressed using $2(n-\ell)$ or fewer explicit covariant derivatives; that is, as a sum of monomials for which $k_{\nabla} \leq 2(n-\ell) . \mathscr{P}_{2 n, 2}$ corsists of quadratic and higher-degree polynomials in $(R, \Omega, E), \mathscr{P}_{2 n, 3}$ of cubic and higher, and so on. We have

$$
\mathscr{P}_{2 n}=\mathscr{P}_{2 n, 1} \supseteq \mathscr{P}_{2 n, 2} \supseteq \cdots \supseteq \mathscr{P}_{2 n, n} ; \quad \mathscr{P}_{2 n, \ell}=0, \quad \ell>n .
$$

An expression which a priori appears only to be in, say, $\mathscr{P}_{6,2}$, may actually be in $\mathscr{P}_{6,3}$; for example, calculating in an orthonormal frame,

$$
\nabla_{i} \nabla_{j} E \cdot \Omega_{i j}=\frac{1}{2}\left[\nabla_{i}, \nabla_{j}\right] E \cdot \Omega_{i j}=\frac{1}{2}\left[\Omega_{i j}, E\right] \cdot \Omega_{i j} .
$$


Using the Bianchi identities for $R$ and $\Omega$, it is easy to show that for $n \geq 1$, the quotient $\mathscr{P}_{2 n} / \mathscr{P}_{2 n, 2}$ is spanned by (the equivalence classes of) the two invariants $\Delta^{n-1} E$ and $\Delta^{n-1} \tau \cdot I$.

Thus we have:

Lemma 1.1. There are universal constants (independent of $(D, M, V)) \quad \alpha_{1}(n)$, $\alpha_{2}(n)$ such that if $n \geq 1$,

$$
e_{n}(x, D)=\alpha_{1}(n) \Delta^{n-1} \tau \cdot I+\alpha_{2}(n) \Delta^{n-1} E+\text { (quadratic and higher). }
$$

Remark 1.2. When applied to $E, \Delta$ is to be understood as the Bochner Laplacian of the bundle End $V$.

Now let $\operatorname{tr}_{V_{x}}=\operatorname{tr}$ be the fiber trace at $x \in M$. Let

$$
a_{n}(x, D)=\operatorname{tr} e_{n}(x, D), \quad a_{n}(D)=\int_{M} a_{n}(x, D)
$$

be the local scalar invariant and global integrated invariant corresponding to $e_{n}(x, D)$. These quantities appear when we take the trace of the asymptotic expansion (1.2), first in $V_{x}$, and then in $L^{2}(V)$ :

$$
\begin{aligned}
& \operatorname{tr} H(t, x, x) \sim(4 \pi t)^{-m / 2} \sum_{n=0}^{\infty} a_{n}(x, D) t^{n}, \\
& \operatorname{Tr}_{L^{2}} e^{-t D}=\int_{M} H(t, x, x) \sim(4 \pi t)^{-m / 2} \sum_{n=0}^{\infty} a_{n}(D) t^{n}, \quad t \downarrow 0 .
\end{aligned}
$$

The last formula shows that $a_{n}(D)$ are spectral invariants of $D$. Since the linear terms in Lemma 1.1 are exact divergences and thus integrate to zero, the leading terms in $a_{n}(D)$ will be quadratic. Thus it is natural to study integrated quadratic terms modulo integrated cubic and higher-degree terms. Again, it is straightforward to show:

Lemma 1.3. If $n \geq 3$, there are universal constants $\beta_{j}(n), j=1, \ldots, 5$, such that

$$
\begin{aligned}
a_{n}(D)=\int_{M} \operatorname{tr} & \left\{\beta_{1}(n)\left|\nabla^{n-2} \tau\right|^{2} I+\beta_{2}(n)\left|\nabla^{n-2} \rho\right|^{2} I+\beta_{3}(n) \nabla^{n-2} \tau \cdot \nabla^{n-2} E\right. \\
& \left.+\beta_{4}(n) \nabla^{n-2} \Omega \cdot \nabla^{n-2} \Omega+\beta_{5}(n) \nabla^{n-2} E \cdot \nabla^{n-2} E\right\} \\
& +(\text { cubic and higher }) . \quad \square
\end{aligned}
$$

Remark 1.4. Formula (1.3) makes sense only for $n \geq 2$. When $n=2$, a sixth invariant, the integral of the norm-squared of the full Riemann tensor $R$, appears. No terms in the derivatives of $R$ appear in (1.3) since, modulo cubic terms and exact divergences, they collapse to linear combinations of the first two invariants. Indeed, integrating by parts and using the Bianchi identity, we get

$$
\int_{M}\left|\nabla^{n-2} R\right|^{2}=\int_{M}\left\{4\left|\nabla^{n-2} \rho\right|^{2}-\left|\nabla^{n-2} \tau\right|^{2}\right\}+\text { (cubic and higher), } \quad n \geq 3 .
$$


If not for the Bianchi identity $d_{\nabla} \Omega=0$, there would be an extra invariant involving $\nabla^{n-3} \nabla_{i} \Omega_{i j} \cdot \nabla^{n-3} \nabla_{k} \Omega_{k j}$. Complete formulas for $a_{n}(x, D)$ are known for $n \leq 3$; see [G1, Theorem 4.3].

For the ordinary scalar Laplacian in dimension $m=2$ (where $\rho=\tau g / 2$ identically), Lemma 1.3 yields

$$
a_{n}(\Delta)=\int_{M}\left\{\left(\beta_{1}(n)+\frac{1}{2} \beta_{2}(n)\right)\left|\nabla^{n-2} \tau\right|^{2}+\cdots\right\},
$$

where " $\ldots$ " is an expression involving at most $2 n-6$ explicit covariant derivatives. $\tau$ encodes all curvature information, so our remainder is a polynomial in covariant derivatives of $\tau$. An important point is that after integration by parts, we can arrange things so that $\nabla^{n-3} \tau$ is the highest derivative that appears. Osgood, Phillips, and Sarnak [OPS] computed the quadratic term in $a_{n}(\Delta)$ in dimension $m=2$; in our notation, they showed that

$$
\beta_{1}(n)+\frac{1}{2} \beta_{2}(n)=n(n-1) c_{n},
$$

where

$$
c_{n}=\frac{(-1)^{n}}{2^{n+1} \cdot 1 \cdot 3 \cdots(2 n+1)}=\frac{(-1)^{n} n !}{2(2 n+1) !} .
$$

Thus if $n \geq 3$ and $M$ is a Riemann surface,

$$
a_{n}(\Delta)=\int_{M}\left\{n(n-1) c_{n}\left|\nabla^{n-2} \tau\right|^{2}+\operatorname{polynomial}\left(\tau, \nabla \tau, \ldots, \nabla^{n-3} \tau\right)\right\} .
$$

The important qualitative observation is that the coefficient in question is nonzero. We say that two metrics are isospectral if their associated scalar Laplacians have the same spectrum. Osgood et al showed that isospectral sets of metrics on a Riemann surface are compact in the $C^{\infty}$ topology, modulo gauge equivalence. The variation across conformal structures is controlled using the nonlocal functional determinant; this also provides an initial Sobolev estimate. An inductive scheme involving the local heat invariants is then set up to get the required higher Sobolev estimates. At the $n$th stage, the leading term in $a_{n}(\Delta)$ controls the highest-order derivative; the remaining terms involve lower derivatives which have been controlled at a previous stage.

A generalization of (1.4) was given by Gilkey in [G1, G3]:

Theorem 1.5. Let $c_{n}=(-1)^{n} n ! / 2(2 n+1)$ !. Then:
(a) $\alpha_{1}(n)=-2 n c_{n}$,
(b) $\alpha_{2}(n)=-4(2 n+1) c_{n}$,
(c) $\beta_{1}(n)=\left(n^{2}-n-1\right) c_{n}$,
(d) $\beta_{2}(n)=2 c_{n}$,
(e) $\beta_{3}(n)=4(2 n+1)(n-1) c_{n}$,
(f) $\beta_{4}(n)=2(2 n+1) c_{n}$,
(g) $\beta_{5}(n)=4(2 n+1)(2 n-1) c_{n}$.

In higher dimensions, the moduli space of conformal structures is not nearly so well understood. It is natural therefore, at least for the time being, to restrict our attention to isospectral families of metrics which lie within a conformal 
class. Theorem 1.5 has been used by Brooks, Chang, Perry, and Yang [CY1$3, \mathrm{BPY}]$ to show that in dimension $m=3$, such families are compact modulo gauge equivalence. These results also provide another derivation of the result of Osgood et al without using the functional determinant, provided the isospectral family in question is contained within a conformal class. The crucial qualitative observation is that $\beta_{1}(n)$ and $\beta_{2}(n)$ are nonzero and have the same sign; this gives the control needed for the higher Sobolev estimates. C. Gordon has informed us that she has found nontrivial isospectral deformations within a conformal class in higher dimensions, so the question is one of great interest.

A proof of Theorem 1.5 $(\mathrm{a}, \mathrm{b})$ based on functorial properties of the heat invariants was given earlier by Gilkey [G1, Theorem 4.1]. The very computational and combinatorial proof of Theorem $1.5(\mathrm{c}-\mathrm{g})$ given in [G3] was unsatisfactory in that it offered little insight into how one might proceed with related problems involving, for example, invariants of boundary value problems. In this paper, we shall give a proof of Theorem 1.5 using functorial properties of the heat invariants. Our approach is based on ideas in [BØ1, BØ2]. Using variational properties of the integrated invariants $a_{n}(D)$ derived in [BØ1], Branson and Ørsted showed in $[\mathrm{B} \varnothing 2, \S 3]$ that one can use explicit formulas for the integrated invariants $a_{n}(D)$ to recover the complete unintegrated invariants $a_{n}(x, D)$; in other words, to recover the missing divergence terms. In this paper, we reverse the process and use the linear divergence terms in $a_{n}(x, D)$ to determine the integrated quadratic terms in $a_{n}(D)$.

Let $D_{\varepsilon}$ be a smooth one-parameter family of operators of the form (1.1); all coefficients, including the metric tensor, are allowed to vary. In our invariant formulation, $D_{\varepsilon}=D\left(g_{\varepsilon}, \nabla_{\varepsilon}, E_{\varepsilon}\right)$. A central part of our approach will be an analysis of the variations $\left.(d / d \varepsilon)\right|_{\varepsilon=0}\left\{a_{n}\left(x, D_{\varepsilon}\right)\right\}$ and $(d / d \varepsilon)_{\varepsilon=0}\left\{a_{n}\left(D_{\varepsilon}\right)\right\}$. Especially nice formulas result when the variation of $D$ is a scalar function multiple of $D$ plus a zeroth-order term:

$$
\left.\frac{d}{d \varepsilon}\right|_{\varepsilon=0} D_{\varepsilon}=f D_{0}+Z, \quad f \in C^{\infty}(M), \text { ord } Z=0 .
$$

This is the case, for example, when $D$ is an operator of the form $\Delta+b \tau$, $b \in \mathbb{R}$, acting on scalar densities of the right degree, and our operator variation comes from variation of the metric within a conformal class. In particular, the ordinary scalar Laplacian can be analyzed in this way. We hope that this new proof of Theorem 1.5 will give additional insight into formulas (a-g) and lead to suitable generalizations, perhaps enabling one to compute the cubic terms in $a_{n}(D)$.

\section{FUNCTORIAL PROPERTIES OF THE HEAT INVARIANTS}

To compute the $\alpha_{i}(n)$, we need to derive some recursion relations. We proceed formally; the necessary analytic justification for the steps involved can be found in [G2, $\mathrm{B} \varnothing 1]$. Let $\theta$ be the usual periodic parameter on the unit circle, and let $h(\theta)$ be a real-valued periodic function. Let $A=d / d \theta+h$; the 
formal adjoint of $A$ is $A^{*}=-d / d \theta+h$. Let

$$
\begin{aligned}
& D_{1}=A^{*} A=\left(-d^{2} / d \theta^{2}\right)-h^{\prime}+h^{2}, \\
& D_{2}=A A^{*}=\left(-d^{2} / d \theta^{2}\right)+h^{\prime}+h^{2} .
\end{aligned}
$$

The leading symbols of the $D_{i}$ are given by the standard (flat) metric on the circle, and the associated connections are trivial. Our bundle endomorphisms are $E\left(D_{1}\right)=h^{\prime}-h^{2}$ and $E\left(D_{2}\right)=-h^{\prime}-h^{2}$. Since the $D_{i}$ are scalar operators, $e_{n}\left(x, D_{i}\right)=a_{n}\left(x, D_{i}\right)$.

Lemma 2.1. (a) Let $(M, g)$ be the standard circle $\left(S^{1}, d \theta^{2}\right)$, and let $D_{i}$ be as above. Let $T=(d / d \theta)(d / d \theta+2 h)$. Then

$$
(2 n-1)\left\{a_{n}\left(x, D_{1}\right)-a_{n}\left(x, D_{2}\right)\right\}=T a_{n-1}\left(x, D_{1}\right), \quad n \geq 1 .
$$

(b) Let $(M, g)$ be a Riemann surface, and let $\Delta_{p}=\delta d+d \delta$ be the p-form Laplacian. Then

$$
n\left\{2 a_{n+1}\left(x, \Delta_{0}\right)-a_{n+1}\left(x, \Delta_{1}\right)\right\}=-\Delta a_{n}\left(x, \Delta_{0}\right), \quad n \geq 0 .
$$

Proof. We use the argument given in $[\mathrm{G} 1, \S 2]$ to prove $(\mathrm{a})$. Let $\left(\operatorname{ker} D_{1}\right)^{\perp}$ be the orthogonal complement to the null space of $D_{1}$ in $L^{2}\left(S^{1}\right)$. Let $\left\{\varphi_{\nu}, \lambda_{\nu}\right\}$ be a spectral resolution of $D_{1}$ on $\left(\operatorname{ker} D_{1}\right)^{\perp}$; then $\left\{A \varphi_{n} / \sqrt{\lambda_{\nu}}, \lambda_{\nu}\right\}$ is a spectral resolution of $D_{2}$ on $\left(\operatorname{ker} D_{2}\right)^{\perp}$. If we differentiate the diagonal values of the heat kernel with respect to $t$, only the nonzero eigenvalues are relevant:

$$
\begin{aligned}
& \frac{\partial}{\partial t} H\left(t, x, x, D_{1}\right)=-\sum_{\lambda_{\nu} \neq 0} \lambda_{\nu} e^{-t \lambda_{\nu}} \varphi_{\nu}^{2}(x), \\
& \frac{\partial}{\partial t} H\left(t, x, x, D_{2}\right)=-\sum_{\lambda_{\nu} \neq 0} e^{-t \lambda_{\nu}}\left(A \varphi_{\nu}\right)^{2}(x) .
\end{aligned}
$$

Let $\Sigma^{\prime}=\Sigma_{\lambda_{\nu} \neq 0}$. Since $D_{1} \varphi_{\nu}=\lambda_{\nu} \varphi_{\nu}$,

$$
\begin{aligned}
\frac{\partial}{\partial t}\{ & \left.H\left(t, x, x, D_{1}\right)-H\left(t, x, x, D_{2}\right)\right\} \\
& =\Sigma^{\prime} e^{-t \lambda_{\nu}}\left\{\left(A \varphi_{\nu}\right)^{2}-\left(D_{1} \varphi_{\nu}\right) \varphi_{\nu}\right\} \\
& =\Sigma^{\prime} e^{-t \lambda_{\nu}}\left\{\varphi_{\nu}^{\prime \prime} \varphi_{\nu}+h^{\prime} \varphi_{\nu}^{2}+\left(\varphi_{\nu}^{\prime}\right)^{2}+2 h \varphi_{\nu}^{\prime} \varphi_{\nu}\right\} \\
& =\frac{1}{2} \Sigma^{\prime} e^{-t \lambda_{\nu}} T\left(\varphi_{\nu}^{2}\right) \\
& =\frac{1}{2} T\left\{H\left(t, x, x, D_{1}\right)\right\} .
\end{aligned}
$$

The null space of $D_{1}$ does not contribute to the last expression because $T\left(\varphi^{2}\right)=$ $2(\varphi A \varphi)^{\prime}$. To complete the proof of $(\mathrm{a})$, we compare the two resulting asymptotic expansions term by term:

$$
\begin{aligned}
& \frac{\partial}{\partial t}\left\{H\left(t, x, x, D_{1}\right)-H\left(t, x, x, D_{2}\right)\right\} \\
& \sim \frac{1}{\sqrt{4 \pi}} \sum_{n=0}^{\infty}\left(n-\frac{1}{2}\right) t^{n-\frac{3}{2}}\left(a_{n}\left(x, D_{1}\right)-a_{n}\left(x, D_{2}\right)\right),
\end{aligned}
$$




$$
\frac{1}{2} T H\left(t, x, x, D_{1}\right) \sim \frac{1}{2 \sqrt{4 \pi}} \sum_{n=0}^{\infty} t^{n-\frac{1}{2}} T a_{n}\left(x, D_{1}\right), \quad t \downarrow 0 .
$$

A similar argument of McKean and Singer [MS, §6] proves (b): let $M$ be a Riemann surface. Let $\left\{\left(\psi_{\nu}, \lambda_{\nu}\right) \mid \nu \in \mathbb{N}\right\}$ be a complete spectral resolution of $\Delta_{0} ; \psi_{0}=$ constant spans the null space of $\Delta_{0}$. If $*$ is the Hodge operator, $\left\{\left(* \psi_{\nu}, \lambda_{\nu}\right) \mid \nu \in \mathbb{N}\right\}$ is a complete spectral resolution of $\Delta_{2}$. Since $\delta+d$ intertwines $\Delta_{0}+\Delta_{2}$ and $\Delta_{1}$,

$$
\left\{\left(d \psi_{\nu} / \sqrt{\lambda_{\nu}}, \lambda_{\nu}\right) \mid \nu \in \mathbb{Z}^{+}\right\} \cup\left\{\left(\delta * \psi_{\nu} / \sqrt{\lambda_{\nu}}, \lambda_{\nu}\right) \mid \nu \in \mathbb{Z}^{+}\right\}
$$

is a complete spectral resolution of $\Delta_{1}$ on $\left(\operatorname{ker} \Delta_{1}\right)^{\perp}$. Since $*$ is a bundle isometry and $\delta=-* d *$,

$$
\begin{aligned}
\frac{\partial}{\partial t}\{\operatorname{tr} & \left.\left.H\left(t, x, x, \Delta_{0}\right)+H\left(t, x, x, \Delta_{2}\right)-H\left(t, x, x, \Delta_{1}\right)\right)\right\} \\
& =\sum_{\nu=1}^{\infty} e^{-t \lambda_{\nu}}\left\{-\lambda_{\nu}\left(\psi_{\nu}^{2}+\left|* \psi_{\nu}\right|^{2}\right)+\left|d \psi_{\nu}\right|^{2}+\left|\delta * \psi_{\nu}\right|^{2}\right\} \\
& =\sum_{\nu=1}^{\infty} e^{-t \lambda_{\nu}}\left\{-2\left(\Delta_{0} \psi_{\nu}\right) \psi_{\nu}+2\left|d \psi_{\nu}\right|^{2}\right\} \\
& =-\Delta \sum_{\nu=0}^{\infty} e^{-t \lambda_{\nu}} \psi_{\nu}^{2} .
\end{aligned}
$$

The key point in the last equality is that $\Delta_{0}$ annihilates $\psi_{0}^{2}$ as well as $\psi_{0}$, so we can include the $\nu=0$ term. Term-by-term comparison of the resulting asymptotic expansions now gives the result.

We shall use Lemma 2.1 to prove $(\mathrm{a}, \mathrm{b})$ of Theorem 1.5. Note that in the setting of Lemma 2.1(a),

$$
\begin{gathered}
\Delta^{n-1} h=(-1)^{n-1} h^{(2 n-2)}, \quad n \geq 1 ; \\
a_{n}\left(x, D_{1}\right)-a_{n}\left(x, D_{2}\right)=2(-1)^{n-1} \alpha_{2}(n) h^{(2 n-1)}+\cdots, \quad n \geq 1 ; \\
T a_{n-1}\left(x, D_{1}\right)=(-1)^{n-2} \alpha_{2}(n-1) h^{(2 n-1)}+\cdots, \quad n \geq 2,
\end{gathered}
$$

where the terms indicated by $\cdots$ are quadratic and higher-degree. This shows that $\alpha_{2}(n)=-\alpha_{2}(n-1) / 2(2 n-1)$ when $n \geq 2$. Working over any vector bundle, the special case in which $E$ is a constant immediately gives $\alpha_{2}(1)=1$, so $\alpha_{2}(n)=-4(2 n+1) c_{n}$ by induction.

If $M$ is a Riemann surface, $E\left(\Delta_{1}\right)=-\tau I / 2$ by the Weitzenböck formula. Thus the two sides of (2.1) are

$$
\begin{aligned}
n\left\{2 a_{n+1}\left(x, \Delta_{0}\right)-a_{n+1}\left(x, \Delta_{1}\right)\right\} & =-n \alpha_{2}(n+1) \operatorname{tr}\left(\Delta^{n} E\left(x, \Delta_{1}\right)\right)+\cdots \\
& =n \alpha_{2}(n+1) \Delta^{n} \tau+\cdots
\end{aligned}
$$

and

$$
-\Delta a_{n}\left(x, \Delta_{0}\right)=-\alpha_{1}(n) \Delta^{n} \tau+\cdots
$$


This shows that $\alpha_{1}(n)=-n \alpha_{2}(n+1)=4 n(2 n+3) c_{n+1}=-2 n c_{n}$.

Integrating (2.1), we see that $2 a_{n}\left(\Delta_{0}\right)-a_{n}\left(\Delta_{1}\right)=0$ for $m=2, n \neq 1$. Recalling Lemma 1.3, this implies that if $n \geq 3$,

$$
\begin{gathered}
0=\int_{M} \operatorname{tr}\left\{\beta_{3}(n) \nabla^{n-2} \tau \cdot \nabla^{n-2} E\left(x, \Delta_{1}\right)+\beta_{4}(n) \nabla^{n-2} \Omega\left(x, \Delta_{1}\right) \cdot \nabla^{n-2} \Omega\left(x, \Delta_{1}\right)\right. \\
\left.+\beta_{5}(n) \nabla^{n-2} E\left(x, \Delta_{1}\right) \cdot \nabla^{n-2} E\left(x, \Delta_{1}\right)\right\}+(\text { cubic and higher }),
\end{gathered}
$$

since the $\beta_{1}(n)$ and $\beta_{2}(n)$ terms from (1.3) cancel. As above, $E\left(x, \Delta_{1}\right)=$ $-\tau I / 2$; since $\tau=2 R_{1212}$,

$$
\operatorname{tr}\left(\nabla^{n-2} \Omega\left(x, \Delta_{1}\right) \cdot \nabla^{n-2} \Omega\left(x, \Delta_{1}\right)\right)=-\left|\nabla^{n-2} R\right|^{2}=-\left|\nabla^{n-2} \tau\right|^{2} .
$$

This proves:

Lemma 2.2. $\beta_{3}(n)+\beta_{4}(n)-\frac{1}{2} \beta_{5}(n)=0$.

We can get all the further relations we need by considering operators of the form $D=\Delta+b \tau$ on $C^{\infty}(M)$, where $b$ is a real parameter. This is the heart of the argument. In this setting, $\Omega=0$ and $E=-b \tau$, so that

$$
\begin{gathered}
a_{n}(x, D)=\left(\alpha_{1}(n)-b \alpha_{2}(n)\right) \Delta^{n-1} \tau+\text { (quadratic and higher) }, \\
a_{n}(D)=\left\{\beta_{1}(n)-b \beta_{3}(n)+b^{2} \beta_{5}(n)\right\} \int_{M}\left|\nabla^{n-2} \tau\right|^{2} \\
+\beta_{2}(n) \int_{M}\left|\nabla^{n-2} \rho\right|^{2}+\text { (cubic and higher) }
\end{gathered}
$$

Now vary the metric through a one-parameter conformal family $g_{\varepsilon}=e^{2 \varepsilon f} g_{0}$, where $f \in C^{\infty}(M)$ and $\varepsilon$ is a real parameter.

Lemma 2.3. Let $D$ be an operator of the form $\Delta+b \tau$ on scalar functions, and let $k(m, b)=\frac{1}{2}(m-2)-2(m-1) b$. For the conformal variation just described,

(a) $\left.(d / d \varepsilon)\right|_{\varepsilon=0} \tau(\varepsilon)=-2 f \tau+2(m-1) \Delta f$,

(b) $\left.(d / d \varepsilon)\right|_{\varepsilon=0} \rho(\varepsilon)=(2-m) \nabla \nabla f+(\Delta f) g$,

(c) $\left.(d / d \varepsilon)\right|_{\varepsilon=0} D_{\varepsilon}=-2 f D+\frac{1}{2}(m-2)[D, f]-k(m, b) \Delta f$,

(d) $\left.(d / d \varepsilon)\right|_{\varepsilon=0} a_{n}\left(D_{\varepsilon}\right)=\int_{M} f\left\{(m-2 n) a_{n}(x, D)+k(m, b) \Delta a_{n-1}(x, D)\right\}$.

Here all quantities and operators on the right-hand sides are calculated in the initial metric $g_{0}$, and the $f$ in the commutator in part (c) is to be viewed as a multiplication operator.

Proof. (a) and (b) are straightforward calculations; see, e.g., [B, (1.27)]. (c) follows from (a) and the conformal covariance of the conformal Laplacian, or Yamabe operator $Y=\Delta+(m-2) \tau / 4(m-1)$ :

$$
\left.\frac{d}{d \varepsilon}\right|_{\varepsilon=0} Y_{\varepsilon}=-2 f Y+\frac{m-2}{2}[Y, f] \text {; }
$$


see, e.g., [BØ1, (1.2)]. (d) is equation (4.1) of the paper [BØ1], which is largely devoted to proving formulas of this type for a very general class of operators having nice conformal properties. In the special case of the conformal Laplacian $Y$, this formula was also derived in [DK, (45),(46)] and [PR, Theorem 3.1]. $Y$ was also used by Gilkey in $[\mathrm{G} 1, \S 4]$ in a recursive calculation of the $a_{n}(x, D)$ when $n \leq 3$ for a general $D$ of the form (1.1). For the sake of completeness, we outline the argument of [BØ1]; the required hard-analytic justification of the steps carried out formally here can be found in $\S 3$ of that paper.

A generalization of a formula of Ray and Singer [RS] gives

$$
\left.\frac{d}{d \varepsilon}\right|_{\varepsilon=0} \operatorname{Tr}_{L^{2}} e^{-t D_{\varepsilon}}=-t \cdot \operatorname{Tr}_{L^{2}}\left\{\left(\left.\frac{d}{d \varepsilon}\right|_{\varepsilon=0} D_{\varepsilon}\right) e^{-t D}\right\} .
$$

When we substitute the operator variation (c) into this formula, the commutator term makes no contribution; this can be seen in three different ways. (1) By working on a bundle of scalar densities of the right degree, we can make the commutator disappear from formula (c) without changing the heat invariants. (2) $-t \cdot \operatorname{Tr}_{L^{2}}\left\{[D, f] e^{-t D}\right\}$ is, by another application of the Ray-Singer formula, the first variation in the $L^{2}$ heat operator trace for the isospectral family of operators $e^{-\varepsilon f} D_{0} e^{\varepsilon f}$. (3) The infinitely smoothing character of the heat operator provides the analytic justification for cyclic permutation of operators under the trace:

$$
\operatorname{Tr}_{L^{2}}\left(D f e^{-t D}\right)=\operatorname{Tr}_{L^{2}}\left(f e^{-t D} D\right)=\operatorname{Tr}_{L^{2}}\left(f D e^{-t D}\right) .
$$

Setting $F=k(m, b) \Delta f$, we have

$$
\begin{aligned}
\left.\frac{d}{d \varepsilon}\right|_{\varepsilon=0} \operatorname{Tr}_{L^{2}} e^{-t D_{\varepsilon}}= & t \cdot \operatorname{Tr}_{L^{2}}\left\{(2 f D+F) e^{-t D}\right\} \\
= & -2 t \frac{\partial}{\partial t}\left\{\operatorname{Tr}_{L^{2}} f e^{-t D}\right\}+t \cdot \operatorname{Tr}_{L^{2}} F e^{-t D} \\
\sim & (4 \pi)^{-m / 2} \sum_{n=0}^{\infty} t^{(2 n-m) / 2} \int_{M}\left\{(m-2 n) f a_{n}(x, D)\right. \\
& \left.+F a_{n-1}(x, D)\right\}, \quad t \downarrow 0 .
\end{aligned}
$$

On the other hand,

$$
\left.\left.\frac{d}{d \varepsilon}\right|_{\varepsilon=0} \operatorname{Tr}_{L^{2}} e^{-t D_{\varepsilon}} \sim(4 \pi)^{-m / 2} \sum_{n=0}^{\infty} t^{(2 n-m) / 2} \frac{d}{d \varepsilon}\right|_{\varepsilon=0} a_{n}\left(D_{\varepsilon}\right) .
$$

Part (d) now follows since, integrating by parts,

$$
\int_{M} F a_{n-1}(x, D)=\int_{M} k(m, b) f \Delta a_{n-1}(x, D) .
$$

Remark 2.4. Both Lemma 2.3(a) and (2.4) are more familiar in their finite, as opposed to infinitesimal, form: if $\bar{g}=e^{2 f} g$, where $f \in C^{\infty}(M)$, then

$$
\Delta u+\frac{m-2}{4(m-1)} \tau u=\frac{m-2}{4(m-1)} \bar{\tau} u^{\frac{m+2}{m-2}}, \quad m \geq 3, \quad \text { where } u=e^{\frac{m-2}{2} f} .
$$


This is called the Yamabe equation. When $m=2,(2.5)$ is replaced by the Gauss curvature prescription equation

$$
\Delta f+\frac{1}{2} \tau=\frac{1}{2} \bar{\tau} e^{2 f} .
$$

( $\frac{1}{2} \tau$ is the Gauss curvature.) In either case,

$$
\bar{Y}=e^{(-(m+2) / 2) f} Y e^{((m-2) / 2) f} ;
$$

this is easily seen using (2.5) or (2.6) together with the conformal deformation law for $\Delta=\delta d$, which in turn is easily obtained from the characterization of $\delta$ as the formal adjoint of $d$. In fact, (2.5) is (2.7) applied to the constant function 1 ; note that the $e^{(m-2) f / 2}$ on the right in $(2.7)$ is to be interpreted as a multiplication operator. Replacing $f$ by $\varepsilon f$ in (2.5), (2.6), and (2.7) and differentiating, we get Lemma 2.3(a) and (2.4).

We now combine (2.2), (2.3), and Lemma 2.3 to finish the proof of Theorem 1.5. By Lemma 2.3(a,b), (2.3), the Bianchi identity div $\rho=\frac{1}{2} \nabla \tau$, and integration by parts, we have

$$
\begin{aligned}
& \left.\frac{d}{d \varepsilon}\right|_{\varepsilon=0} \int_{M}\left|\nabla^{n-2} \tau\right|^{2}=4(m-1) \int_{M} f\left\{\Delta^{n-1} \tau+\cdots\right\}, \\
& \begin{aligned}
\left.\frac{d}{d \varepsilon}\right|_{\varepsilon=0} & \int_{M}\left|\nabla^{n-2} \rho\right|^{2}=m \int_{M} f\left\{\Delta^{n-1} \tau+\cdots\right\}, \\
\left.\frac{d}{d \varepsilon}\right|_{\varepsilon=0} a_{n}\left(D_{\varepsilon}\right) & \\
= & \int_{M} f\left\{\left[4(m-1)\left(\beta_{1}(n)-b \beta_{3}(n)+b^{2} \beta_{5}(n)\right)+m \beta_{2}(n)\right] \Delta^{n-1} \tau+\cdots\right\},
\end{aligned}
\end{aligned}
$$

where the terms indicated by ... are quadratic and higher-degree. But by Lemma 2.3(d) and (2.2),

$$
\begin{aligned}
\left.\frac{d}{d \varepsilon}\right|_{\varepsilon=0} a_{n}\left(D_{\varepsilon}\right)=\int_{M} f\{[( & m-2 n)\left(\alpha_{1}(n)-b \alpha_{2}(n)\right) \\
& \left.\left.+k(m, b)\left(\alpha_{1}(n-1)-b \alpha_{2}(n-1)\right)\right] \Delta^{n-1} \tau+\cdots\right\} .
\end{aligned}
$$

This yields the fundamental identity

$$
\begin{aligned}
4(m-1)\left\{\beta_{1}(n)-b \beta_{3}(n)+b^{2} \beta_{5}(n)\right\}+m \beta_{2}(n) \\
\quad=(m-2 n)\left(\alpha_{1}(n)-b \alpha_{2}(n)\right)+k(m, b)\left(\alpha_{1}(n-1)-b \alpha_{2}(n-1)\right) .
\end{aligned}
$$

Because this is a polynomial equation in $(m, b)$ good for $m=1,2,3, \ldots$ and $b \in \mathbb{R}$, it holds identically. Evaluating at $(m, b)=(0,0)$ and using the fact that $c_{n-1}=-2(2 n+1) c_{n}$, we get the desired formula for $\beta_{1}(n)$. Evaluating at $(m, b)=(1,0)$, we get $\beta_{2}(n)$. Taking $\partial / \partial b$ at $(m, b)=(2,0)$ gives $\beta_{3}(n)$, and taking $\partial^{2} / \partial b^{2}$ anywhere but at $m=1$ gives $\beta_{5}(n)$. Lemma 2.2 then gives the desired value of $\beta_{4}(n)$, and the proof of Theorem 1.5 is complete. 


\section{REMARKS}

3.1. As a check on our calculations, suppose that $M$ has a spin structure, and let $V$ be the full spinor bundle of fiber dimension $\mu=2^{[m / 2]}$. Let $D$ be the square of the Dirac operator. By the Lichnerowicz formula, $E=-\tau I / 4$, and by [BØ2, pp. 98,99] and analogous calculations with $\nabla^{n-2}$ operating on each curvature quantity,

$$
\operatorname{tr} \nabla^{n-2} \Omega \cdot \nabla^{n-2} \Omega=-\frac{\mu}{8}\left|\nabla^{n-2} R\right|^{2}, \quad n \geq 2 .
$$

By [BØ1, last formula on p. 278], the conformal variations of the integrated heat invariants are given by

$$
\left.\frac{d}{d \varepsilon}\right|_{\varepsilon=0} a_{n}(D)=(m-2 n) \int_{M} f a_{n}(x, D) .
$$

This yields

$$
\begin{gathered}
(m-1)\left\{4 \beta_{1}(n)-\beta_{3}(n)+\frac{1}{4} \beta_{5}(n)+\frac{1}{2} \beta_{4}(n)\right\}+m\left\{\beta_{2}(n)-\frac{1}{2} \beta_{4}(n)\right\} \\
=(m-2 n)\left(\alpha_{1}(n)-\frac{1}{4} \alpha_{2}(n)\right), \quad n \geq 3 .
\end{gathered}
$$

Subtracting this from the $b=\frac{1}{4}$ version of $(2.8)$, we get

$$
\frac{1}{2} \beta_{4}(n)=-\frac{1}{2}\left(\alpha_{1}(n-1)-\frac{1}{4} \alpha_{2}(n-1)\right)=(2 n+1) c_{n} .
$$

3.2. Our analysis of the operators $A^{*} A$ and $A A^{*}$ on the circle (Lemma 2.1(a)) could be replaced by consequences of another conformal principle: let $Y$ be the conformal Laplacian as before, and consider the conformal deformation of Lemma 2.3. Suppose $m$ is even. Then there is a formula for the local conformal variation of the heat invariant $a_{(m-2) / 2}(x, Y)$ :

$$
\left.\frac{d}{d \varepsilon}\right|_{\varepsilon=0} a_{\frac{m-2}{2}}(x, Y)=(2-m) a_{\frac{m-2}{2}}(x, Y) .
$$

Equation (3.1) was first observed somewhat indirectly: an analogous result holds for an invariant appearing in Hadamard's asymptotic expansion of the Green's function for the conformal d'Alembertian on a Lorentz manifold. This was noted in connection with investigations into Huygens' principle; see, e.g., [S, $\S 4$; W, (3.11)]; the invariant in question is the "first log term" in Hadamard's expansion. One can establish (3.1) using this result, plus: (1) a correspondence between Hadamard invariants of the Green's functions for the conformal d'Alembertian in the Lorentz category and the conformal Laplacian in the Riemannian category, and (2) a correspondence between the Hadamard and heat invariants for $Y$ in the Riemannian category. (1) is a question of analytic continuation in signature for local invariants; see, e.g. [BØ2, §7] for a careful handling of this. (2) is also part of a proof given by Parker and Rosenberg [PR, Theorem 2.1], which is more direct in that it takes place entirely within Riemannian geometry. We shall present an outline of this proof, which replaces (1) by a "first log term" argument in the Riemannian category. 
In even dimensions $m \geq 4$, in a neighborhood of the diagonal in $M \times M$, the Green's function $G(x, y, D)$ of any $D=\Delta+b \tau$ admits an expansion

$$
\begin{array}{r}
G(x, y, D)=(4 \pi)^{-m / 2}\left\{\sum_{n=0}^{(m-4) / 2} \Gamma\left(\frac{m}{2}-n-1\right) a_{n}(x, y, D)\left(\frac{r}{2}\right)^{2-m+2 n}\right. \\
\left.-a_{\frac{m-2}{2}}(x, y, D) \log r^{2}+O\left(r^{0}\right)\right\},
\end{array}
$$

where $r=r(x, y)$ is the geodesic distance from $y$ to $x$, and $O\left(r^{0}\right)$ and the $a_{n}(x, y, D)$ are smooth two-point functions with $a_{n}(x, x, D)=a_{n}(x, D)$. In fact, $a_{n}(x, y, D)$ is the Hadamard-Minakshisundaram-Pleijel two-point function [H, MP], obtained by inductive solution of a sequence of transport equations.

Under a finite conformal change of metric $\bar{g}=e^{2 f} g, f \in C^{\infty}(M)$, the conformal covariance law (2.7) shows that the Green's function changes by

$$
\bar{G}(x, y, Y)=G(x, y, \bar{Y})=e^{-\frac{m-2}{2}(f(x)+f(y))} G(x, y, Y) .
$$

Thus our infinitesimal conformal change has the effect

$$
\left.\frac{d}{d \varepsilon}\right|_{\varepsilon=0} G(x, y, Y)=-\frac{m-2}{2}(f(x)+f(y)) G(x, y, Y) .
$$

But by [BØ1, Lemma 5.1], $\left.(d / d \varepsilon)\right|_{\varepsilon=0} r^{2}=2 \tilde{f} r^{2}$, where $\tilde{f}$ is the average value of $f$ on the $g$-geodesic from $y$ to (nearby) $x$. Replacing all terms in (3.2) by sufficiently high Taylor polynomials in normal coordinates (so that all error terms are subsumed into the $O\left(r^{0}\right)$ term), applying (3.3), and evaluating at $x=y$, one easily gets $(3.1)$.

Now one can get partial information about the $a_{n}(x, D)$ for $n \geq 1$, that is, about the universal coefficients $\alpha_{i}(n)$ and $\beta_{j}(n)$, by looking at $(3.1)$ in dimension $m=2 n+2$. Here $E=-n \tau / 2(2 n+1)$, so Lemma $2.3(a)$ immediately gives $\alpha_{1}(n)=n \alpha_{2}(n) / 2(2 n+1)$ for $n \geq 1$. Together with our argument based on the two-dimensional de Rham complex (Lemma 2.1(b), which gives $\left.\alpha_{1}(n)=-n \alpha_{2}(n+1)\right)$, this is enough information to generate all of the $\alpha_{i}(n)$. Taking this approach, our calculation of the $\alpha_{i}(n)$ and $\beta_{j}(n)$ uses only the two-dimensional de Rham complex and operators of the form $\Delta+b \tau$ on scalar functions.

In fact, the consequences of (3.1) for the determination of the heat invariants are far more powerful than just this; we expect that (3.1) will be one of the most useful functorial properties in an eventual calculation of the integrated cubic terms in the $a_{n}(D)$. 
3.3. For bounded domains in $\mathbb{R}^{2}$, Melrose [M] has shown that the relevant coefficients of leading terms in the heat invariants, including those depending on the geometry of the boundary, are nonzero. A partial analysis of variational formulas like Lemma $2.3(\mathrm{~d})$ for boundary-value problems in higher dimensions is given in [BØ3, §2]. Given [CY1-3, BPY], it seems reasonable to hope that the compactness question for sets of isospectral domains in $\mathbb{R}^{3}$ is approachable at this point.

\section{REFERENCES}

[B] T. Branson, Differential operators canonically associated to a conformal structure, Math. Scand. 57 (1985), 293-345.

[BØ1] T. Branson and B. Ørsted, Conformal indices of Riemannian manifolds, Compositio Math. 60 (1986), 261-293.

[ВØ2] _ Conformal deformation and the heat operator, Indiana Univ. Math. J. 37 (1988), 83-110.

[BØ3] __ Conformal geometry and global invariants, preprint.

[BPY] R. Brooks, P. Perry, and P. Yang, Isospectral sets of conformally equivalent metrics, Duke Math. J. 58 (1989), 131-150.

[CY1] S.-Y. A. Chang and P. C. Yang, Isospectral conformal metrics on 3-manifolds, J. Amer. Math. Soc. 3 (1990), 117-145.

[CY2] _ Compactness of isospectral conformal metrics on $S^{3}$, Comment. Math. Helv. 64 (1989), 363-374.

[CY3] _ The conformal deformation equation and isospectral sets of conformal metrics, Contemp. Math. (to appear).

[DK] J. Dowker and G. Kennedy, Finite temperature and boundary effects in static space-times, J. Phys. A 11 (1978), 895-920.

[G1] P. Gilkey, Recursion relations and the asymptotic behavior of the eigenvalues of the Laplacian, Compositio Math. 38 (1979), 201-240.

[G2] Invariance theory, the heat equation, and the Atiyah-Singer index theorem, Publish or Perish, Wilmington, Delaware, 1984.

[G3] Leading terms in the asymptotics of the heat equation, Contemp. Math. 73 (1988), 79-85.

[H] J. Hadamard, Le problème de Cauchy et les èquations aux dérivées partielles linéaires hyperboliques, Hermann et Cie, Paris, 1932.

[MS] H. P. McKean, Jr. and I. M. Singer, Curvature and the eigenvalues of the Laplacian, J. Differential Geom. 1 (1967), 43-69.

[M] R. Melrose, Isospectral sets of drumheads are compact in $C^{\infty}$, preprint.

[MP] S. Minakshisundaram and $\AA$. Pleijel, Some properties of the eigenfunctions of the Laplace operator on Riemannian manifolds, Canad. J. Math. 1 (1949), 242-256.

[OPS] B. Osgood, R. Phillips, and P. Sarnak, Compact isospectral sets of surfaces, J. Funct. Anal. 80 (1988), 212-234.

[PR] T. Parker and S. Rosenberg, Invariants of conformal Laplacians, J. Differential Geom. 25 (1987), 199-222.

[RS] D. B. Ray and I. M. Singer, R-torsion and the Laplacian on Riemannian manifolds, Adv. in Math. 7 (1971), 145-210. 
[S] R. Schimming, Lineare Differentialoperatoren zweiter Ordnung mit metrischem Hauptteil und die Methode der Koinzidenzwerte in der Riemannschen Geometrie, Beitr. z. Analysis 15 (1981), 77-91.

[W] V. Wünsch, Konforminvariante Variationsprobleme und Huygenssches Prinzip, Math. Nachr. 120 (1985), 175-193.

Mathematical Institute, University of Copenhagen, Universitetsparken 5, 2100 Copenhagen $\varnothing$, Denmark

Current address, Thomas P. Branson: Department of Mathematics, The University of IOWA, IOWA CITY, IOWA 52242

Current address, Peter B. Gilkey: Department of Mathematics, University of Oregon, EUGENE, OREGON 97403

Current address, Bent Ørsted: Department of Mathematics and Computer Science, Odense University, Campusvej 55, 5230 Odense M, Denmark 